\title{
Quantifying the dispersion quality of partially aggregated colloidal dispersions by high frequency rheology
}

\section{Journal Article}

Author(s):

Schroyen, Bram; Swan, James W.; Van Puyvelde, Peter; Vermant, Jan

Publication date:

2017-11

Permanent link:

https://doi.org/10.3929/ethz-b-000207876

Rights / license:

In Copyright - Non-Commercial Use Permitted

Originally published in:

Soft Matter 13(43), https://doi.org/10.1039/C7SM01690E

Funding acknowledgement:

157147 - Exploiting particle shape and connectivity to interrogate flocculated suspension mechanics. (SNF) 


\title{
Journal Name
}

\section{ARTICLE TYPE}

Cite this: DOI: 10.1039/xxxxxxxxxx

Accepted Date

DOI: 10.1039/xxxxxxxxxx

www.rsc.org/journalname

\section{Quantifying the dispersion quality of partially aggre- gated colloidal dispersions by high frequency rheol- ogy}

\author{
Bram Schroyen $^{a, b}$, James W. Swan ${ }^{c}$, Peter Van Puyvelde ${ }^{b}$ and Jan Vermant ${ }^{* a}$
}

An important parameter for the performance of nanomaterials is the degree by which the nanoparticles are dispersed in a matrix. Optical microscopy or scattering methods are useful to characterise the state of dispersion, but are not generally applicable to all materials. Electron microscopy methods are laborious in preparation and typically offer only quantitative information on a very local scale. In the present work we investigate how high frequency rheological measurements can be used for partially dispersed suspensions at intermediate to higher particle loadings, even for high viscous matrices. Although the contribution of the particles is particularly visible in the low frequency linear viscoelastic behaviour, a more direct relationship between rheological properties and degree of dispersion can be derived from the loss modulus in the high frequency limit. To this end, a home-built piezo shear rheometer is constructed to extend the frequency range typically accessible by commercial rotational rheometers. Measurements on spherical silica particles, with a varying degree of dispersion in low molecular weight PDMS, are used to demonstrate how high frequency rheometry can be used to quantify dispersion quality. The linear viscoelastic properties are compared to analytical scaling theories to demonstrate that a hydrodynamically dominated regime is reached. The dependence of the relative high frequency loss modulus on volume fraction is then compared to predictions of a hydrodynamic viscosity model for the derivation of a dispersion quality index. It is used to follow the evolution of the dispersion quality as a function of mixing time and consumed power.

\section{Introduction}

Considerable efforts have been devoted to the design and processing of nanomaterials to exploit novel functionalities associated with the nanoscale. Inorganic particles have been used to improve the physical properties of materials such as ceramics, elastomers or polymer films ${ }^{1-3}$. More recent applications include the usage in construction materials, e.g. to improve concrete durability, or in biomedical applications and advanced drug delivery ${ }^{4,5}$. The final properties of nanocomposites depend on the characteristics of particles and matrix and their interactions. However, an additional factor that strongly affects the material performance is the degree of filler dispersion achieved during mixing ${ }^{6}$. For instance in coating and printing applications, high degrees of pigment dispersion are crucial for the final product

\footnotetext{
${ }^{a}$ Department of Materials, ETH Zurich, Vladimir-Prelog-Weg 5, 8093 Zurich, Switzerland. E-mail: jan.vermant@mat.ethz.ch

${ }^{b}$ Department of Chemical Engineering, KU Leuven, Celestijnenlaan 200f, 3001 Heverlee, Belgium

${ }^{b}$ Department of Chemical Engineering, MIT, 77 Massachusets Avenue, Cambridge, MA 02139
}

quality 7,8 . The performance of polymer nanocomposites is greatly influenced by the dispersion quality as well ${ }^{9,10}$. The mechanical properties of clay-polymer nanocomposites for example are found to increase with increasing degree of dispersion due to a higher degree of exfoliation of the clay platelets ${ }^{11}$.

Due to the colloidal nature of nanoparticles, dispersion methods need to overcome the van der Waals or other attractive forces, necessitating complex or energy-intensive processes ${ }^{12,13}$. Having efficient tools to monitor the dispersion quality during processing are hence worthwhile ${ }^{14}$. However, quantifying the degree of dispersion is not always trivial. Electron microscopy offers sufficient resolution in the nanoscale, but sampling and sample preparation are often laborious and it is not always possible or cost effective to carry out sufficient measurements to obtain statistically relevant data. Furthermore, not all liquid dispersions can be solidified or used in cryo-preparation. Scattering methods offer statistically averaged data, but samples may either have too much or too little contrast. Depending on the particle size distribution, any $q$-dependent analysis can be strongly influenced by outliers in the aggregate size distribution. 
For turbid systems advances have been made using multiple scattering techniques, either removing the multiple scattered light in techniques such as 3D-dynamic light scattering or fully exploiting it in diffusing wave spectroscopy ${ }^{15-17}$. But still these techniques are performant only when the nanocomposites fulfil certain constraints, regarding for example scattering contrast, ergodicity and light absorption. An alternative is to turn our attention to methods which probe the dispersion quality indirectly, for example looking at macroscopic mechanical properties such as the viscosities or linear viscoelastic properties. Earlier work has illustrated the potential of rheological methods to assess the dispersion quality in either polymeric matrices $11,18,19$ or low viscous resins ${ }^{20,21}$. Linear viscoelastic properties are very sensitive to changes in the microstructure. Moreover, the use of rheology and small amplitude oscillatory measurements in particular allows to study dispersions in the undiluted fluid state, even up to very high concentrations, without interfering with the dispersion process or state, hence offering potential for in-situ monitoring. The potential of tuning the frequency of the oscillation makes it possible to vary the length scale on which the structure is excited, from large scale structures at low frequencies, to local phenomena at high frequencies.

Information on the dispersion state from rheological data is often inferred qualitatively from the low frequency elastic modulus $G^{\prime}$ as its behaviour is mainly dictated by the particle contribution $10,18,20,22$. However, this contribution is not straightforward to analyse quantitatively without a priori knowledge on the interactions and on the details of the large scale structures formed, ranging from the packing of aggregates to the fractal dimensions of sample spanning networks ${ }^{23,24}$. Low frequency measurements probe the collective microstructure and are much more influenced by thixotropic and ageing effects ${ }^{25}$. At higher frequencies, however, the observed structural length scale becomes localised, eventually to the scale of the colloidal building blocks which make up the dispersion ${ }^{26}$. Under certain conditions, the high frequency limit may be dominated by the hydrodynamic contributions, which are purely dissipative and will be different from the limit set by well dispersed individual particles ${ }^{27,28}$. In this region the individual building blocks can be probed even when being part of a global network which allows for a quantitative approach to interrogate the nature of the local scale microstructure of the dispersion ${ }^{11,20}$. In case of spherical fillers, the presence of aggregates or agglomerates leads to an increase in the effective volume fraction due to the particles being packed in inefficient structures ${ }^{29}$. The influence is less trivial for anisotropic particles as an improved dispersion quality can lead to larger aspect ratios and increased frictional interactions ${ }^{11,22}$.

In order to probe the high frequency limit, the time scale of the applied oscillation must be much smaller than the time scale of diffusive colloidal motion ${ }^{30}$. For Brownian hard spheres, the relevant frequency range is given by 26,31

$$
\frac{k_{B} T}{a^{3} 12 \pi^{2} \eta_{m}} \ll f \ll \frac{\eta_{m}}{2 \pi \rho_{m} a^{2}},
$$

with $f$ the frequency in $\mathrm{Hz}, a$ the particle radius, $k_{B} T$ the thermal energy scale ${ }^{23}$ and $\eta_{m}$ and $\rho_{m}$ on both sides the matrix viscosity and density, respectively. The upper limit of the hydrodynamic regime is when fluid and particle inertial contributions come in to play and when hydrodynamic interactions can no longer be considered to act instantaneously. Both effects occur at a similar frequency, depending on the respective densities of particles and matrix ${ }^{26}$. In case of hard spheres of $50 \mathrm{~nm}$ radius dispersed in a Newtonian matrix of $0.1 \mathrm{~Pa} \cdot \mathrm{s}$, the resulting frequency range is 10 $\mathrm{Hz} \ll f \ll 10^{9} \mathrm{~Hz}$. Colloidal interactions alter the force balance in Eq. 1, for instance due to an increase of the effective radius at contact stemming from repulsive interactions ${ }^{32}$. In the presence of attractive interactions, the characteristic time scale was found to scale with the range of the interaction potential through the thermodynamic stresses and the structure's response to flow ${ }^{33}$. In addition the hydrodynamic properties of the aggregates will be different from those of the individual particles ${ }^{34,35}$.

The frequency dependence of the high frequency moduli reveals in a subtle manner the interplay between the colloidal and hydrodynamic interactions, and their effects on the nonequilibrium microstructure. In particular, the behaviour depends on the relative size of the hydrodynamic lubrication layer with respect to the range of the structural interaction forces. For hard spheres the discontinuity in the potential at contact leads to a limiting high frequency storage modulus which scales as $f^{1 / 227}$, which was observed experimentally by van der Werff and coworkers ${ }^{36,37}$. When near field lubrication hydrodynamic interactions come into play, for example for particles with softer interaction potentials, a Maxwellian behaviour with a limiting high frequency modulus has been observed ${ }^{38}$. The high frequency rheology can be further influenced by the presence of attractive interactions, and both direct contributions of the interaction force as well as changes in the hydrodynamic contributions are introduced as the particle spacing changes $26,27,32,33$. It was observed that the existence of both repulsive and attractive colloidal interactions can shift the entry into the high frequency region towards higher frequencies and alter the asymptotic behaviour towards the hydrodynamic limit ${ }^{32,33,39}$. Experimentally, in the hydrodynamic high frequency limit, the curves of the loss moduli are expected to become parallel with that of the suspending medium.

Conventional rheometers are limited in their frequency range until $\sim 20-50 \mathrm{~Hz}$ due to instrument inertia. An extension of this limit for high frequency rheometry is required in order to probe the high frequency regime for most suspensions ${ }^{40}$. However, going to very high frequencies is also not of interest, as the penetration depth $\delta$ of the shear wave should be such that bulk properties are probed ${ }^{41}$. The penetration depth depends on the frequency $f$ as well as on the density $\rho$ and complex moduli of 
the sample :

$$
\delta=\frac{\left|G^{*}\right|}{2 \pi f} \sqrt{\frac{2}{\rho} \cdot \frac{1}{\left|G^{*}\right|-G^{\prime}}} .
$$

For instance for a $0.1 \mathrm{~Pa} \cdot \mathrm{s}$ Newtonian matrix measured at 1 $\mathrm{MHz}$, the penetration depth $\delta \approx 6 \mu \mathrm{m}$. In order to measure bulk properties of colloidal dispersions consisting of colloidal particles and aggregates, the operating frequency should be kept $<100$ $\mathrm{kHz}$. Torsional resonator methods ${ }^{42,43}$, operating at discrete frequencies between $1-100 \mathrm{kHz}$, are highly sensitive and have been used for determining the shear properties of wormlike micelles ${ }^{44}$ or measuring the colloidal charge in low viscous dispersions ${ }^{30}$. However, they require a relatively large sample volume and the use of discrete resonances hampers to study frequency dependent scalings. In addition, dealing with more viscous fluids remains challenging which limits the applicability for nanocomposite materials. Subresonant piezorheometers can be used to characterise both viscoelastic fluids and solids at continuous frequency ranges $<10 \mathrm{kHz}^{40}$. For instance, piezorheometers have been applied for the characterisation of concentrated colloidal dispersions and colloidal gels ${ }^{45,47}$, wormlike micellar solutions ${ }^{46}$ or colloid-liquid crystal composites ${ }^{50}$. Shear rheometers are of specific interest due to their simple flow profile and were developed for both torsional ${ }^{48}$ or linear flow $^{49,50 .}$

In the present work, high frequency rheometry is proposed as a technique to quantify the degree of dispersion of partially dispersed colloidal suspensions, for intermediate to high volume fractions. A piezo shear rheometer (PSR) is constructed, which operates using a linear shear motion ${ }^{49,50}$. In order to create samples of varying states of dispersion, spherical nanoparticles are dispersed in a low viscous matrix by different mixing techniques and mixing times. The dispersion quality is quantified by subjecting the high frequency rheological data to a hydrodynamic analysis, after having identified the relevant scaling regime. As an application the evolution of the dispersion state during mixing is monitored by using the rheological dispersion quality index. Note that in this work, the term "aggregate" is used for the building blocks of the dispersion created during the dispersion process. Although the size of these building blocks depends on the intensity and duration of mixing, and describing them according to the IUPAC nomenclature as "agglomerates" may be more appropriate, the assumption in the measurement is that the linear response regime is probed and the structure is not affected by the measurement.

\section{Experimental}

\section{Preparation of silica dispersions}

Dispersions of colloidal silica particles in a low viscous polydimethylsiloxane (PDMS) matrix (350 cSt Silicone oil, Sigma-Aldrich) were used as model systems. Partially hydrophobic silica particles were kindly provided by Nissan Chemical (MEK-ST-ZL, Nissan Chemical Industries, Ltd.) as a $30.7 \mathrm{wt} \%$ stock suspension in MEK. Multi-angle dynamic light scattering (DLS) measurements (ALV/CGS-3 Goniometer, ALV-Gmbh)
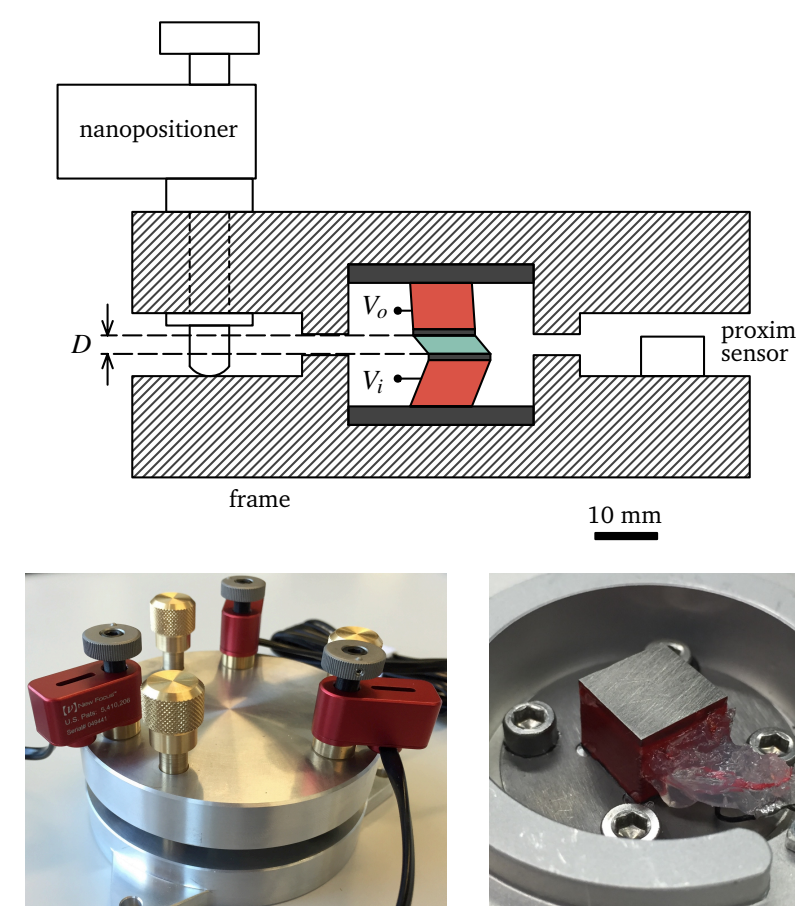

Fig. 1 Top : schematic of the piezorheometer. Bottom : pictures of the frame with nanopositioners and fixation screws (left) and of the excitation piezo inside the measurement cell (right).

provided an average particle size of $d_{h}=130 \mathrm{~nm}$, which was confirmed by SEM pictures.

Batches of $10 \mathrm{~g}$ were prepared with particle concentrations varying between 4.4 and $22.2 \mathrm{vol} \%$. PDMS was added to a stock suspension of silica particles in MEK and mixed. This procedure was used to avoid the creation of large and irreversible aggregates during drying. The sample was placed in a Rotavap (R-214 Rotavapor, Büchi) at controlled conditions of $243 \mathrm{mbar}$ and $50^{\circ} \mathrm{C}$ to evaporate MEK near its critical point. Sample families of varying dispersion quality were prepared by mixing for 15 min with different techniques of increasing energy input. Particles were dispersed by magnetic stirring at $750 \mathrm{rpm}(\mathrm{S})$ as to create a low intensity, global mixing. Note that at concentrations higher than $15 \mathrm{vol} \%$, the samples were solid-like prior to mixing so that magnetic stirring was no longer possible. High shear mixing (UT) was employed as a second method by means of an Ultra-Turrax disperser (Ultra-Turrax T25 S25N-10G, IKA-Werke GmbH) run at $8000 \mathrm{rpm}$. The highest dispersing intensity was obtained by subjecting the samples to ultrasonic pulses (US). A finger shaped sonicator (UP400S, Hielscher) was operated at $60 \%$ intensity and $50 \%$ cycle at a frequency of $24 \mathrm{kHz}$. All samples were stored under vacuum for $15 \mathrm{~min}$ after dispersing to remove entrained air bubbles. Measurements were carried out immediately after this sample preparation to rule out potential effects of sedimentation or ageing. 


\section{High frequency setup}

High frequency oscillatory measurements were performed by means of a piezorheometer. Fig. 1 shows a schematic view of the instrument together with pictures of the frame and measurement cell. The measurement cell consists of two piezo-ceramic stacks (PICA P-141.05 Shear Actuators, PI Ceramic GmbH) that operate in a simple shear mode, similar in design to Roth et al. ${ }^{50}$. Each stack is glued to a stainless steel base plate on one side and a square Ti substrate of $10 \mathrm{~mm}$ side length on the other. The cables have to be well shielded to prevent electronic crosstalk. Samples are placed in between both piezo stacks.

The base plates of both elements are fixed to an aluminium frame, which is used to control the measurement gap and shield off the inner part of the cell from the environment. The alignment tools are installed on the outside of the frame. In order to optimise the alignment accuracy and efficiency, a three-point mechanical alignment method is used ${ }^{51}$. Inductive proximity sensors (SMT-9700 20N Non-contact Displacement Sensors, KAMAN Instrumentation) are installed on the bottom frame, measuring both the gap and parallelism with an accuracy of $10 \mathrm{~nm}$ and maximum range of $400 \mu \mathrm{m}$. The gap is controlled by 3 nano-positioning screws (8301 Piezo Linear Actuator, Newport Corporation) with a minimum step size of $30 \mathrm{~nm}$. The zero-gap was determined by measuring frictional forces between the substrates of both elements during small amplitude oscillations, and the resulting parallelism is $<1 \mu \mathrm{m}$ or $<0.005^{\circ}$. The gap setting is maintained by 3 large screws with ultra-fine thread to ensure a strong coupling and shift the resonance frequencies of the device to higher values.

The piezo-ceramic stack positioned at the bottom (Fig. 1) is used as the excitation unit and is connected to the reference output of a LockIn amplifier (MFLI, Zurich Instruments), which applies a sinusoidal voltage signal $V_{o}(t)$ :

$$
V_{o}(t)=V_{o} \cdot e^{(j 2 \pi f t)} .
$$

As a result of the inverse piezoelectric effect, the sinusoidal output voltage creates a linear shear deformation $z(t)$ at the surface of the bottom substrate. The frequency $f$ can be varied until $\sim 2500 \mathrm{~Hz}$, at which point the setup reaches its first large resonances. The setup is operated in the gap loading limit so that the deformation wave propagates completely through the sample and induces a shear force $F(t)$ on the top substrate directly proportional to the sample properties ${ }^{52}$. This limits the measurement gap $D$, which must be smaller than the shear wavelength $\lambda_{s}$ :

$$
D \ll \lambda_{s}=\frac{1}{f \sqrt{\frac{\rho}{\left|G^{*}\right|}} \cos \left(\frac{\delta}{2}\right)} .
$$

For a low viscous PDMS matrix of $0.34 \mathrm{~Pa} \cdot \mathrm{s}$ measured at $1 \mathrm{kHz}, \lambda_{s}$ equals $\sim 2 \mathrm{~mm}$. In this limit, $F(t)$ has the same frequency as the applied motion but differs in phase. The resulting shear movement of the piezo-ceramic plates generates a voltage signal $V_{i}(t)$ that is recorded by the input of the LockIn amplifier :

$$
V_{i}(t)=V_{i} \cdot\left[e^{j\left(2 \pi f t+\theta_{s}\right)}+e^{-j\left(2 \pi f t+\theta_{s}\right)}\right],
$$

with $\theta_{s}$ the measured phase shift with respect to the reference frequency. Depending on the sample properties the magnitude of the output voltage can be smaller than $1 \mu \mathrm{V}$ so that low filter bandwidths and careful shielding are required.

At frequencies between $20-2000 \mathrm{~Hz}$, the relevant frequency range for extending the commercial rotational rheometers, no other contributions (inertia, resonance) were measured so that sample properties could be determined directly from the input $V_{i}(t)$. The amplitude ratio $F / z$ and phase angle $\delta$ are calculated as

$$
\begin{aligned}
\frac{F}{z} & =\frac{V_{i}}{V_{o}} \cdot \frac{C}{d_{15}^{2}}, \\
\delta & =\theta_{s},
\end{aligned}
$$

from which the complex moduli can be derived. In Eq. 6, $d_{15}=(5.4 \pm 0.3) \cdot 10^{-9} \mathrm{~m} / \mathrm{V}$ is the shear deformation coefficient and $C=(2.8 \pm 0.6) \cdot 10^{-8} \mathrm{~F}$ the electric capacitance of the piezoceramic stacks. Note that at a gap $D=200 \mu \mathrm{m}$, which is well below the shear wavelength (Eq. 4), and output magnitude $V_{o}=10$ $\mathrm{V}$, the resulting shear deformation amplitude and strain amplitude are $53 \mathrm{~nm}$ and $0.027 \%$, respectively. Fig. 2 shows $F / z$ and $\delta$ for 3 PDMS grades of different viscosities, measured at a gap of $200 \mu \mathrm{m}$. The measured values are in good agreement with predictions based on the Newtonian (zero shear) viscosity values and illustrate the onset of viscoelastic responses at higher frequencies, where the slope of the amplitude ratio starts to decrease and the phase angle deviates from $90^{\circ}$. The sensitivity limit of the amplitude ratio is determined from the random noise value on the input of the LockIn amplifier and equals $(F / z)_{\min } \approx 7.1 \sqrt{f} \mathrm{~m} / \mathrm{V}$. The upper limit is set by the compliance of the piezo-ceramic stack relative to the sample. The low frequency range of the PSR overlaps with the accessible frequency range of a commercial device. By direct comparison of the measured phase angle with that of a commercial stress-controlled rheometer (DHR-3, TA Instruments - USA), the phase resolution of the PSR was determined to be $\delta_{\text {err }} \sim 2^{\circ}$. The setup is operated at room temperature in a controlled laboratory environment.

\section{Rheological measurements}

Information on the dispersion quality was obtained by performing dynamic rheological measurements within the linear region over an extended frequency range. Commercial rotational stresscontrolled rheometers (MCR 302, Anton Paar - Austria ; DHR-3, TA Instruments - USA) were used for oscillatory experiments in a frequency range between $0.01-20 \mathrm{~Hz}$. In order to compare the measurement data quantitatively with the high frequency results, tests were performed with a $40 \mathrm{~mm}$ diameter plate plate geometry at $200 \mu \mathrm{m}$ gap distance. A pre-shear of $25 \mathrm{~s}^{-1}$ was applied for $300 \mathrm{~s}$ after which the sample was allowed to recover for $300 \mathrm{~s}$, until a reference structure at rest was obtained. High frequency 

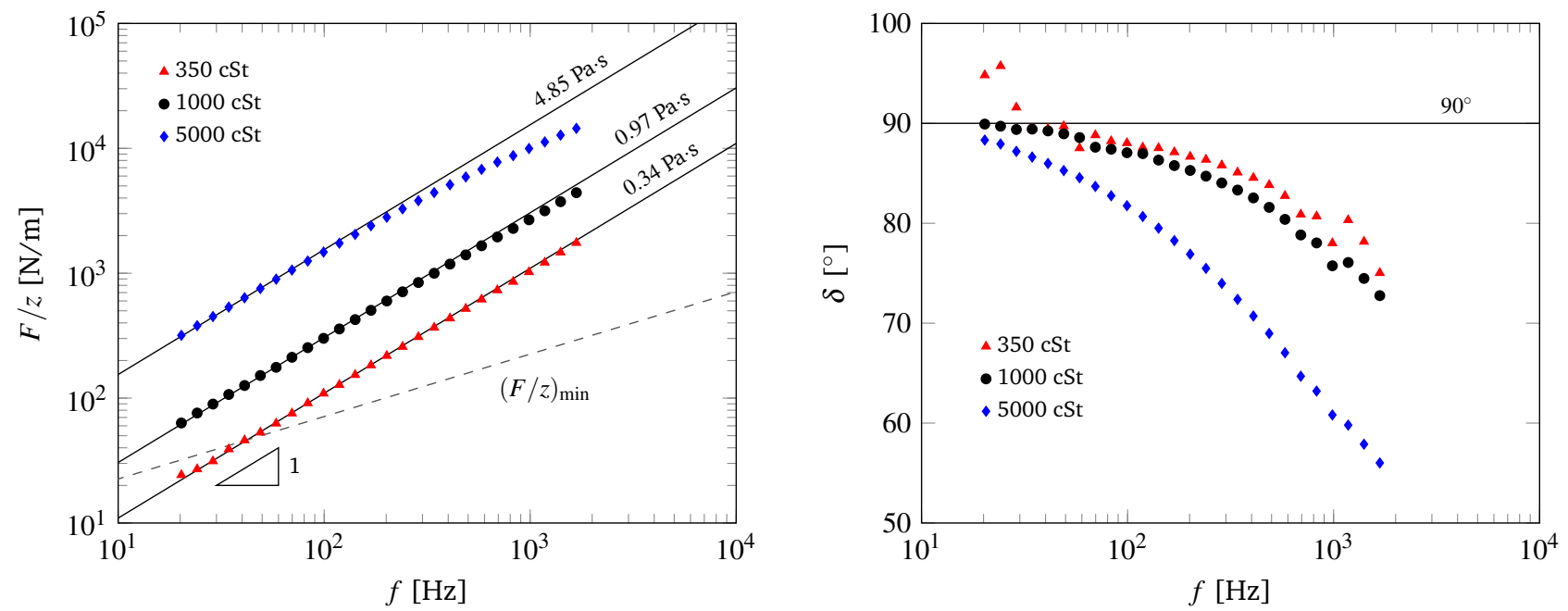

Fig. 2 Measurements of three PDMS grades of different viscosities, showing the frequency dependence of the amplitude ratio between force and displacement (left) and phase angle (right). The solid lines are the low frequency Newtonian limits. The dashed line on the amplitude ratio graph represents the lower sensitivity limit.

measurements were carried out with the piezo shear rheometer (PSR) at frequencies between $20-2000 \mathrm{~Hz}$. The operating gap was kept at $200 \mu \mathrm{m}$. A resting period of $300 \mathrm{~s}$ was applied after loading before starting measurements. All measurements were carried out at room temperature.

\section{Monitoring a dispersion process}

The evolution of the dispersion state during high shear UT mixing was monitored at rotor speeds of 4000, 8000 and $12400 \mathrm{rpm}$. Samples were taken at discrete moments in time for high frequency rheological measurements. In addition, multi-angle DLS measurements were performed to determine the equilibrium state of the aggregates after intensive mixing. The dispersions were diluted to 0.01 vol\% and subjected to gentle stirring in order to homogenise the samples without influencing the dispersion state. Measurements were carried out at angles ranging from $20^{\circ}-90^{\circ}$ with a $5^{\circ}$ angle step size and a measurement time of $500 \mathrm{~s}$ per angle to obtain sufficient data averaging. Sedimentation was not observed over the course of the measurements. The mean aggregate size was determined by fitting the autocorrelation curves as a function of the decay time $\tau$ with a cumulant expansion :

$$
g_{2}(\tau)-1=b\left[e^{-c \tau}+\left(\frac{d}{2} \tau^{2}\right)\right]^{2}
$$

The parameter $b$ is defined by the intercept whereas $c$ and $d$ contain information on the average decay $\bar{\Gamma}$ and polydispersity, respectively.

\section{Results and discussion}

\section{Scaling with frequency}

The data obtained with the rotational and the piezo rheometer for samples produced by UT mixing are shown in Fig. 3 for three silica volume fractions and pure PDMS. The measurements are representative of samples obtained via other dispersion methods as well. Due to the large matrix viscosity, the linear viscoelastic response is largely dominated by the hydrodynamic interactions among the particles. At high frequencies, the magnitude of the loss modulus is proportional to that of the suspending PDMS matrix, as shown in earlier work ${ }^{24}$. The loss moduli are plotted in Fig. 3 (left) for the UT samples, showing a general increase with increasing volume fraction. When judging the quality of rheological data, it is insightful to look directly at the phase angle, shown at the right in Fig. 3. At low frequencies, the angle deviates form $90^{\circ}$. This deviation is more pronounced with increasing volume fraction and reflects contributions to the stress from relaxation of stresses among large aggregates. At the highest frequencies measured with the rotational device the effects of instrument inertia lead to minor deviations (not shown here). For the PSR the phase angle at low frequencies is not very precise due to signal to noise issues. At high frequencies, the matrix viscoelasticity dictates the phase angle in the suspensions, and the effect of particle volume fraction on the phase angle is negligible.

The loss modulus normalised by the matrix contribution: $G_{r e l}^{\prime \prime}=G^{\prime \prime} / G_{m}^{\prime \prime}$, shows more clearly how contributions to viscoelasticity from colloidal and hydrodynamic interactions change with concentration and frequency (Fig. 4). At low frequencies, the formation of large aggregates or a volume spanning network structure causes $G_{r e l}^{\prime \prime}$ to increase significantly with volume fraction, which is also seen in Fig. 3. The relative loss moduli level off at high frequencies to a constant value, however. Evolution of $G_{r e l}^{\prime \prime}$ with frequency shows the value of the extended frequency range in the PSR. The low frequency behaviour stems from a complex interplay between colloidal and hydrodynamic interactions, whereas at high frequencies the constant value of the moduli can be attributed to a purely hydrodynamical regime, where only the effective volume fraction of the aggregates and 

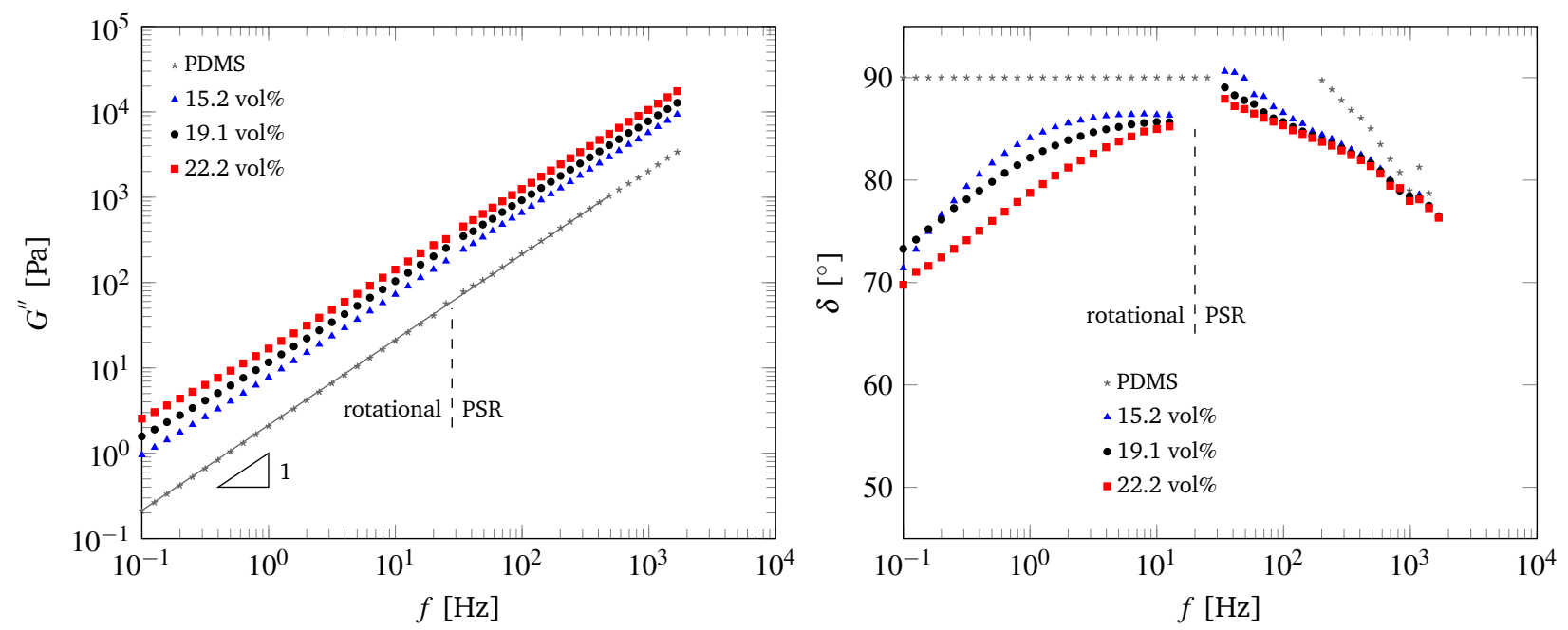

Fig. 3 Linear viscoelastic loss moduli (left) and phase angles (right) of the UT samples measured at different volume fractions, compared to the matrix modulus.

inter-aggregate structure matter. The relative high frequency modulus $G_{r e l, h f}^{\prime \prime}$, calculated as the average value in the HF limit (Fig. 4), will be used for further analysis to characterise the dispersion quality ${ }^{28}$.

Varga and Swan presented calculations of the stress response of a semi-dilute colloidal dispersion in the presence of attractive depletion interactions. Whereas their calculations are a virial expansion in the particle volume fraction, and hence not necessarily valid up to the volume fractions studied here, it is useful to compare their predictions and scalings to the experimentally observed quantities. They observed a transition between two viscoelastic regimes: one dominated by colloidal interactions among the par-

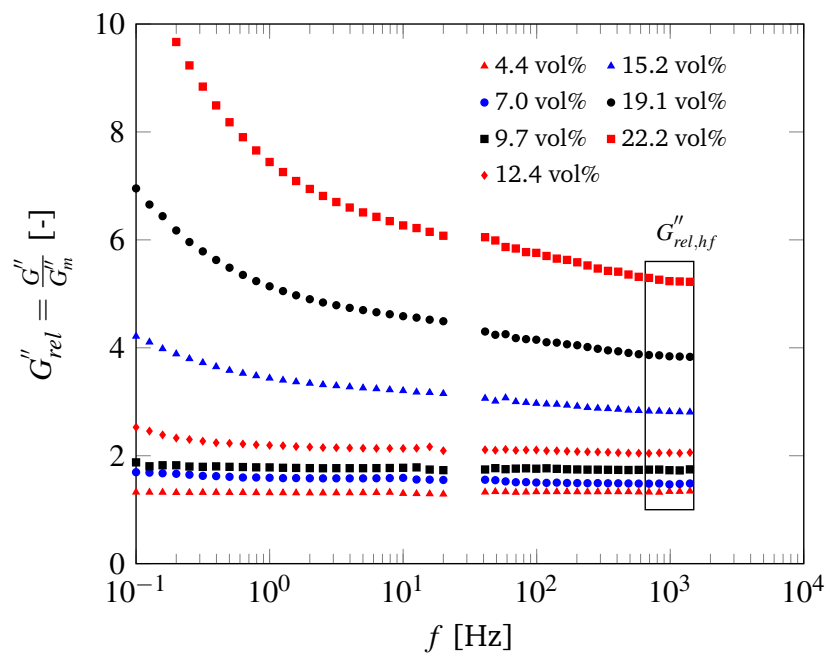

Fig. 4 Relative loss moduli $G_{r e l}^{\prime \prime}=\frac{G^{\prime \prime}}{G_{m}^{\prime \prime}}$ of the UT samples at different concentrations. High frequency moduli (closed symbols) are again compared with moduli obtained from a commercial device (open symbols). The box indicates the frequency window used for the calculation of $G_{r e l, h f}^{\prime \prime}$. ticles, and the other at high frequencies, which is dominated by hydrodynamic interactions. The hydrodynamic regime exhibits a particular power law behaviour at high frequencies for the reduced real part of the complex viscosity:

$$
\eta_{r}^{\prime}=\frac{\eta^{\prime}-\eta_{h f}^{\prime}}{\eta_{0}^{\prime}-\eta_{h f}^{\prime}}
$$

where $\eta_{0}^{\prime}$ and $\eta_{h f}^{\prime}$ respectively are the low frequency and high frequency limits of $\eta^{\prime}$. This quantity can be used to detect the emergence of the hydrodynamically dominated regime. Because the suspending PDMS is intrinsically viscoelastic (Fig. 2), a plateau value for $\eta_{h f}^{\prime}$ is not necessarily reached over the measured frequency range. Instead, an equivalent reduced viscosity

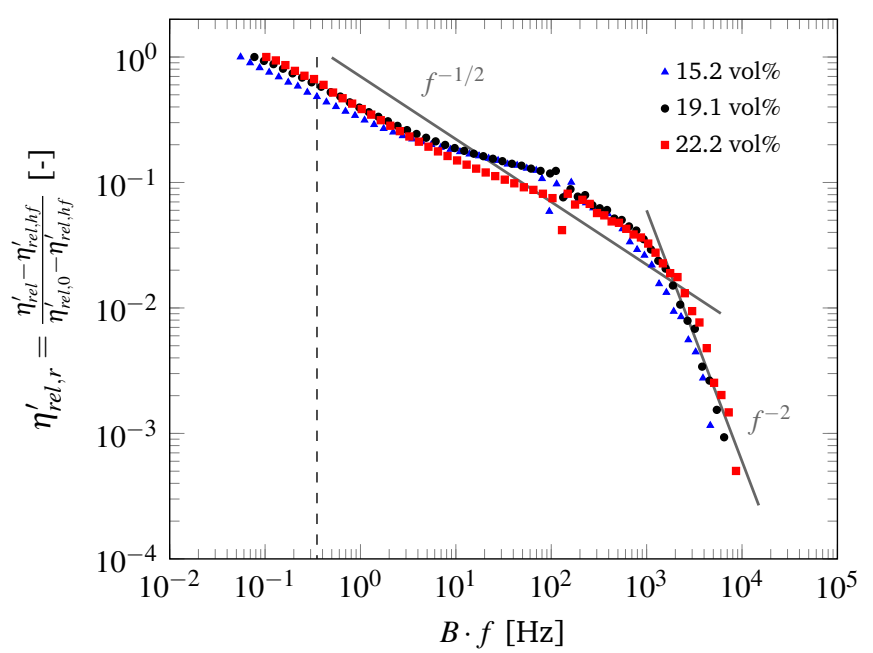

Fig. 5 Real part of the relative complex viscosity reduced by the high and low frequency values as a function of the shifted frequency for 3 different volume fractions of the UT samples. The dashed line represents a rough approximation of the characteristic frequency $f_{c}=0.35 \mathrm{~Hz}$, based on Brownian motion (Eq. 1). Solid lines illustrate the limiting slopes in both regimes. 
for the experimental dispersions is constructed from the relative complex viscosities by replacing $\eta^{\prime}$ in equation 9 with $\eta_{r e l}^{\prime}=G_{r e l}^{\prime \prime}$. This removes the effect of matrix viscoelasticity but still enables examination of frequency dependent variations in the loss modulus resulting from colloidal and hydrodynamic interactions among the suspended particles.

After this substitution, the value of $\eta_{r e l, h f}^{\prime}$ is just the measured relative high frequency modulus $G_{r e l, h f}^{\prime \prime}$ (Fig. 4). The quantity $\eta_{r e l, 0}^{\prime}$ is arbitrarily chosen as the value of $G_{r e l}^{\prime \prime}$ measured at the lowest sampled frequency, as no zero shear viscosity is obtained for the concentrated, strongly attractive suspensions studied here. Yet, this arbitrary choice affects only the magnitude of the numerator without affecting the slopes of the reduced viscosity on a log-log plot. Another useful rescaling results from a consideration of the matrix viscoelasticity. Because the PDMS matrix can be considered to be an isotropic fluid, a simple 'Gleissle' shift factor $B$ can be used to renormalise the frequency ${ }^{53}$. This shift factor reflects the increase of the internal shear rate at constant stress, which in the hydrodynamic regime and well below the relaxation time of the matrix is proportional to the real part of the relative complex viscosity at high frequencies; thus,

$$
B=\eta_{r e l, h f}^{\prime}=G_{r e l, h f}^{\prime \prime} .
$$

Fig. 5 shows the resulting real part of the reduced relative viscosity $\eta_{r e l, r}^{\prime}$ for the 3 highest concentrations as a function of the rescaled frequency, $B \cdot f$. Scaling the reduced relative viscosity and frequency in this way provides a comparative basis for dispersions of different particle loadings and matrix viscoelasticity. The rescaled viscoelastic response of all three suspensions is the same, which can be explained by ther fact that the local aggregate microstructures probed at these high frequencies are similar across a range of primary particle volume fractions ${ }^{27}$.

In Fig. 5 two different scaling regimes can be observed. At frequencies above the characteristic frequency $f_{c}$ (Eq. 1) but below $1 \mathrm{kHz}$, the reduced relative viscosities decay with a power law. A slope of $\sim f^{-1 / 2}$ is shown for reference. This power law scaling suggests relaxation of small deformations in the aggregate microstructure dominated by colloidal interactions. In the second regime, above $1 \mathrm{kHz}$, the power law slope increases in magnitude such that the reduced relative viscosities decay as $f^{-2}$. In this regime, the internal aggregate structure is frozen. Hydrodynamic forces generate micro-structural perturbations among aggregates and their collective diffusion relaxes the resulting entropic stresses ${ }^{32}$. The transition between regimes marks the entry into the high frequency limit of viscoelasticity, in which hydrodynamic forces as opposed to colloidal forces dominate the energy dissipation. The reduced frequency at which this transition happens is expected and observed to be independent of the volume fraction.

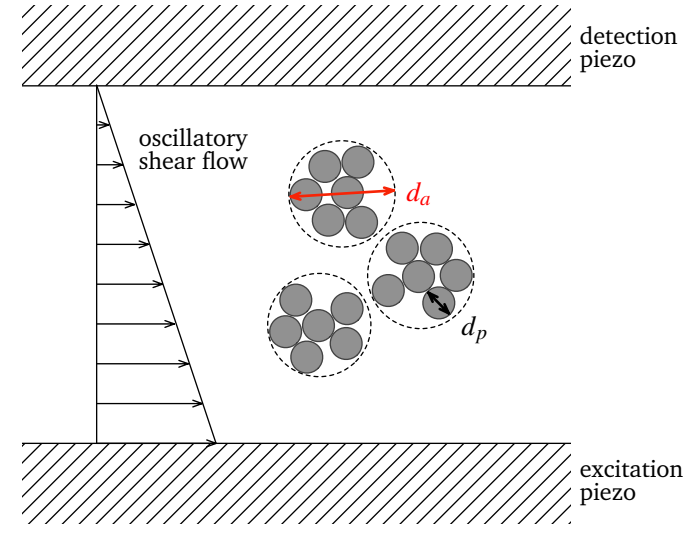

Fig. 6 Schematic of the measurement principle : aggregates of colloidal particles $\left(d_{p} \approx 130 \mathrm{~nm}\right)$ subjected to an oscillatory shear flow in a plate-plate geometry. $d_{a}$ represents the hydrodynamic aggregate size.

\section{Derivation of a dispersion quality index}

A quantification of the degree of dispersion can be determined from the relative loss modulus measured in the hydrodynamic regime. The measurement principle is illustrated in Fig. 6. In the high frequency or hydrodynamic limit, the linear viscoelastic properties are dominated by matrix contributions and by hydrodynamic interactions between the aggregates. The presence of particles distorts fluid streamlines ${ }^{23,54}$. Such distortions of the flow field increase the energy dissipation during flow and hence the high frequency loss modulus. The magnitude of this effect depends on the effective hydrodynamic volume taken up by the partially dispersed silica particles and increases with aggregate size.

Fig. 7 gives the evolution of $G_{r e l, h f}^{\prime \prime}$ as a function of primary particle volume fraction for different dispersing methods, i.e. the $S$, UT and US samples. The degree of dispersion can be quantified

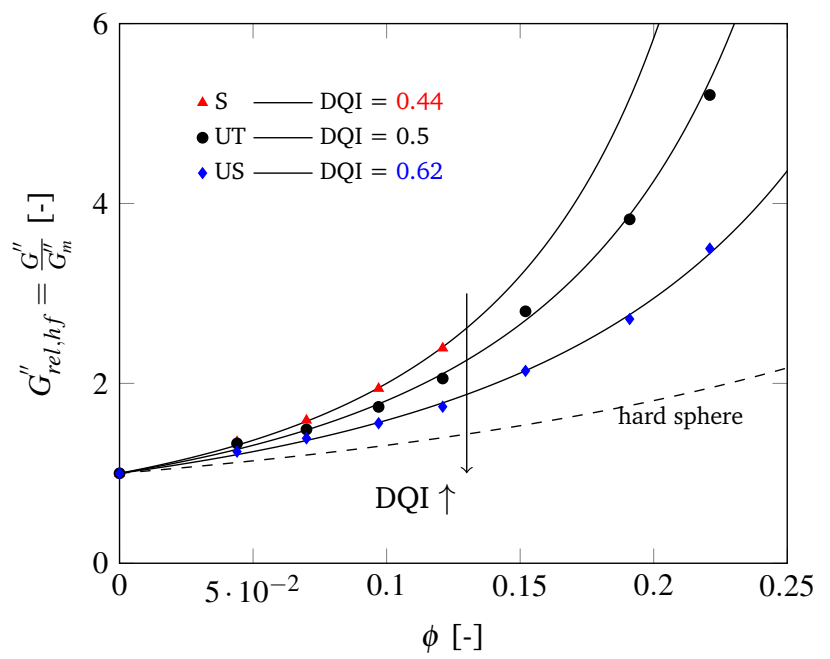

Fig. 7 Evolution of the relative high frequency loss moduli as a function of volume fraction for three different dispersion methods : magnetic stirring (S), high shear mixing (UT) and ultrasonication (US). Solid lines correspond to the fits of Eq. 11 with $\phi_{e f f}$ whereas the dashed line represents the hard sphere case. 
by comparing the measured high frequency viscosity to the high frequency viscosity of a fully dispersed suspension of Brownian hard spheres at the same volume fraction. This quantity is purely hydrodynamic. It reflects only the structure of the suspension and not the relaxation processes resulting from colloidal interactions among primary particles. Different semi-empirical relationships have been derived for describing the viscosity of hard-spheres in the hydrodynamic limit ${ }^{55,56}$; however, the simulations of Sierou and Brady ${ }^{57}$, which provide a complete description of the hydrodynamic interactions incorporating both long-range many-body interactions and near-field lubrication effects, yield the most rigorous data set available. Based on these results an analytical form for the high frequency limit of the relative loss modulus was derived $^{58}$ :

$$
G_{r e l, h f}^{\prime \prime}=\frac{1+\frac{3}{2} \phi_{e f f}\left[1+\phi_{e f f}\left(1+\phi_{e f f}-2.3 \phi_{e f f}^{2}\right)\right]}{1-\phi_{e f f}\left[1+\phi_{e f f}\left(1+\phi_{e f f}-2.3 \phi_{e f f}^{2}\right)\right]},
$$

which is valid for random suspensions of colloidal or nonBrownian suspensions up until volume fractions $\phi_{\text {eff }} \leq 0.56$ or $G_{r e l, h f}^{\prime \prime}<12.6$. The volume fraction $\phi_{\text {eff }}$ is the effective volume fraction based on the hydrodynamic radius of the freely suspended particles. $G_{r e l, h f}^{\prime \prime}$ based on Eq. 11 for $\phi_{e f f}$ equal to the primary particle volume fraction is plotted in Fig. 7 as a dotted line and represents the viscosity of the suspensions in their most dispersed state. When aggregates are present, the effective volume fraction will be higher, and hence the high frequency loss moduli are expected to grow more quickly. This is indeed what is observed in Fig. 7. It can also be observed from Fig. 7 that as the mixing intensity is increased ( $\rightarrow \mathrm{UT} \rightarrow \mathrm{US}$ ), the measured moduli decrease, which is consistent with an improved dispersion quality in response to more vigorous mixing.

For aggregated suspensions, the effective volume fraction can be defined as the ratio of the primary particle volume fraction: $\phi$, to a dimensionless quantity termed the dispersion quality index: DQI,

$$
\phi_{e f f}=\frac{\phi}{\mathrm{DQI}} .
$$

The dispersion quality index indicates the distance from a fully dispersed state in which the high frequency viscosity is well described by equation 11 with $\phi_{e f f}=\phi$ and $\mathrm{DQI}=1$. In principle, DQI varies between 0 and 1 and quantifies the reduction in volume accessible to the suspending media for flow around suspended aggregates. Thus, DQI is a measure of the effect of hydrodynamic interactions among aggregates only. This modification of Eq. 11 results in a single-parameter model for $G_{r e l, h f}^{\prime \prime}$. The measured relative high frequency viscosity is fitted for the DQI for each of the sample families, and the calculated fits are plotted in Fig. 7. The fit models agree well with all the experimental measurements. This implies that the local building blocks of the dispersion remain unchanged, even at higher volume fractions at which these structures interact more strongly (Fig. 3 and 4). The fitting is valid until when the dispersion becomes dense and aggregate formation is hindered. The DQI improves (grows) when increasing the mixing intensity with a value of 0.44

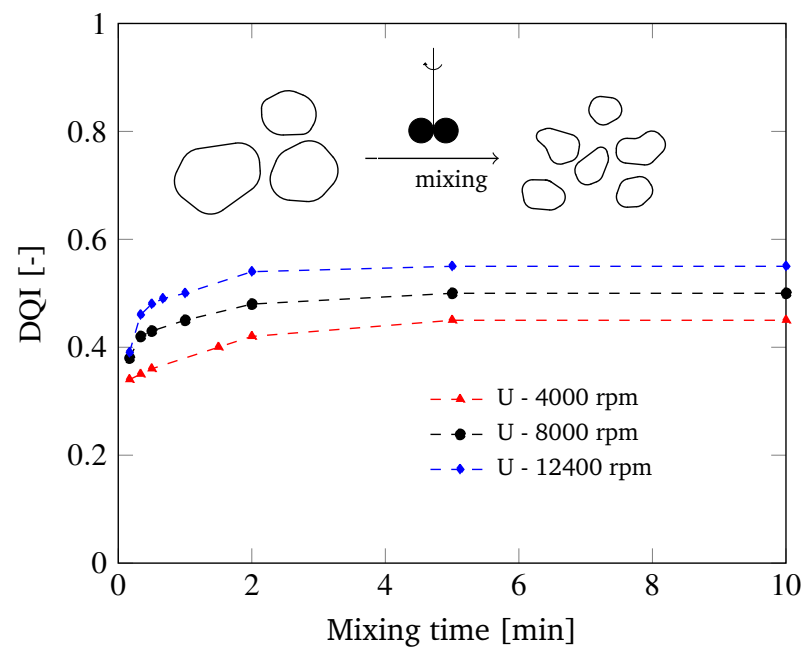

Fig. 8 Evolution of the DQI as a function of mixing time for a dispersion of 15.2 vol\% silica particles. Particles are dispersed by Ultra-Turrax mixing at 3 different intensities.

for simple magnetic stirring and 0.62 for ultrasonicated samples. This confirms that aggregates persist in these suspensions even with high mixing intensities. Note that the absolute value of the effective volume fraction is influenced by the internal aggregate structure $^{59}$. The DQI should be interpreted in relative terms as it probes the global structure of the dispersion and not the internal structure of the aggregates. However, since the proposed index is unaffected by Brownian motion and colloidal interactions, it enables the quantification of the dispersion quality of colloidal suspensions in a well-defined manner.

\section{Monitoring a dispersion process}

Dispersion processes are used to break up larger aggregates into dispersed and uniformly distributed particles. The derived DQI can be used to monitor the evolution of the dispersion state during mixing operation. Fig. 8 displays the evolution of the DQI during high shear mixing as a function of mixing time for different rotor speeds. For all samples, the DQI evolved in time, gradually improving and reaching a terminal, equilibrium value after several minutes. In addition, at all times, the measured DQI increased with increasing mixing intensity. The evolution towards an equilibrium state is somewhat faster with increased mixing intensities, as well. Note that the time resolution of the DQI measurements is restricted by sampling limitations. However, improved sampling rates might be achieved through in-situ measurements. These results are in agreement with previous findings on hydrodynamic stress induced dispersion and fragmentation ${ }^{60}$, which reported that with sufficient mixing, an equilibrium aggregate size emerges at each imposed shear rate.

The equilibrium hydrodynamic aggregate sizes are also calculated from DLS measurements on dispersed and diluted samples via the Stokes-Einstein equation and compared with the equilibrium DQI in Table $1^{60,61}$. The average aggregate size varies between 2-4 times the primary particle diameter. 
Table 1 Equilibrium DQI and average aggregate size $d_{a}$ for different mixing velocities $N$. Primary particles have a diameter $d_{p} \approx 130 \mathrm{~nm}$.

\begin{tabular}{llll}
\hline$N[\mathrm{rpm}]$ & DQI [-] & $d_{a}[\mathrm{~nm}]$ & $N_{p} / N_{a}$ \\
\hline 4000 & 0.45 & 445 & 18.0 \\
8000 & 0.5 & 365 & 11.1 \\
12400 & 0.55 & 275 & 5.21 \\
\hline
\end{tabular}

Both high frequency rheology and DLS measurements show an improvement in the dispersion quality with increasing mixing intensity. Furthermore, DLS results confirm that small aggregates consisting of a few particles continue to exist at high mixing intensities. Note that the cumulant analysis (Eq. 8) assumes a monomodal distribution of spherical aggregates. However, since both high frequency loss moduli and DLS data probe the hydrodynamic aggregate size, results from both methods can be compared ${ }^{28}$. When comparing the results of the two methods in Table 1 , the evolution of the DQI and $d_{a}$ agree with each other. It must however be emphasized that, although the aggregates are assumed to remain unchanged during dilution and homogenisation, changes in size or structure cannot be ruled out completely.

As the dispersion quality index is proportional to the effective hydrodynamic volume fraction of aggregates in the dispersion, it can be used in combination with a measurement of the average aggregate size to determine the average number of primary particles per aggregate: $\mathrm{DQI}=\phi / \phi_{\mathrm{eff}} \approx\left(N_{p} / N_{a}\right)\left(d_{p} / d_{a}\right)^{3}$. From Table 1 , the number of primary particles per aggregate $N_{p} / N_{a}$, decreases linearly with the mixing velocity. When assuming that the aggregates are fractal-like, a power law relationship $N_{p} / N_{a}=\left(d_{a} / d_{p}\right)^{d_{f}}$ suggests an aggregate fractal dimension: $d_{f} \approx 2.25$, which corresponds with a reaction-limited aggregation process. Using these same relations, a priori knowledge of the aggregate fractal dimension and measurement of the DQI can be used to estimate the average aggregate size: $d_{a} / d_{p} \approx \mathrm{DQI}^{1 /\left(d_{f}-3\right)}$. Note that this analysis neglects the polydispersity of the aggregates and assumes a mass-fractal-like structure. This assumption is not necessarily valid, since other types of aggregate structures have been reported for colloidal dispersions as well ${ }^{62,63}$, and the aggregate structure may be altered during the dispersion process. However, for certain systems such analyses can be of practical engineering value for connecting the dimensionless dispersion quality index to a physical dimension in the suspension.

\section{Conclusions}

Rheology is of particular interest for assessing the dispersion state of partially dispersed colloidal particles since it is highly sensitive to the microstructure. Whereas obtaining information about the state of dispersion from the low frequency rheology would necessitate assumptions about the interplay between the microstructure and the colloidal interactions, high frequency rheology offers a more elegant method to quantify the degree of dispersion. For this purpose a homebuilt piezorheometer was constructed that operates in linear shear flow. The mechanical design of the setup, detection method and alignment sensitivity were optimised to perform accurate measurements at frequencies between 20 $2000 \mathrm{~Hz}$. Measurements on model dispersions of colloidal silica particles showed that in the high frequency limit, obtained by the piezorheometer, the loss modulus is dominated by matrix contributions and hydrodynamic interactions. The limiting high frequency modulus $G_{h f}^{\prime \prime}$ was used to assess the dispersion quality by quantification of the distance to the case of fully dispersed particles through a hydrodynamic viscosity model. The resulting dispersion quality index DQI depends only on the increased effective volume fraction stemming from aggregates. In case of spherical particles, this index varies from 0 to 1 with a value of 1 representing finely dispersed particles. Although the proposed method does not account for the internal aggregate structure, it enables to assess the dispersion state of partially dispersed nanoparticles in a well-defined manner. The derived DQI was used to follow the evolution of the average degree of dispersion during mixing with a high shear mixing device. The degree of dispersion was observed to improve with increasing mixing time and intensity.

\section{Acknowledgements}

PVP and BS acknowledge funding of SIM-Flanders (SBO project TRAP), JV acknowledges the European Union (EU) Horizon 2020-INFRAIA-2016-1, EUSMI project no 731019, and the Swiss National Science Foundation, project number 157147. JS and JV acknowledge support of the MISTI Global Seed Fund of MIT. Dr. G. K. Auernhammer (MPI Mainz) is thanked for helpful comments on the design of the PSR, W. Schmidheiny is thanked for his work on the construction of the PSR.

\section{References}

1 W. J. Stark, P. R. Stoessel, W. Wohlleben and A. Hafner, Chem. Soc. Rev., 2015, 44, 5793-5805.

2 Y. Zhang, S. Ge, B. Tang, T. Koga, M. H. Rafailovich, J. C. Sokolov, D. G. Peiffer, Z. Li, A. J. Dias, K. O. McElrath, M. Y. Lin, S. K. Satija, S. G. Urquhart, H. Ade and D. Nguyen, Macromolecules, 2001, 34, 7056-7065.

3 E. L. Cussler, S. E. Hughes, W. J. Ward and R. Aris, J. Membr. Sci., 1988, 38, 161-174.

4 C\&EN, Building Small, 2011.

5 Y. Matsumoto, R. Chen, P. Anikeeva and A. Jasanoff, Nat. Commun., 2015, 6, 8721.

6 D. W. Schaefer and R. S. Justice, Macromolecules, 2007, 40, 8501.

7 D. M. Yebra and C. E. Weinell, in Advances in Marine Antifouling Coatings and Technologies, Woodhead Publishing, 2009, ch. Key issues in the formulation of marine antifouling paints.

8 C\&EN, Fit to Print, 2002.

9 Y. Dzenis, Science, 2008, 319, 419.

10 S. K. Kumar, N. Jouault, B. Benicewicz and T. Neely, Macromolecules, 2013, 46, 3199-3214.

11 J. Vermant, S. Ceccia, M. K. Dolgovskij, P. L. Maffettone and C. W. Macosko, J. Rheol., 2007, 51, 429-450.

12 M. E. Mackay, A. Tuteja, P. M. Duxbury, C. J. Hawker, B. Van Horn, Z. Guan, G. Chen and R. S. Krishnan, Science, 
2006, 311, 1740.

13 B. Stuyven, Q. Chen, W. Van de Moortel, H. Lipkens, B. Caerts, A. Aerts, L. Giebeler, B. Van Eerdenbrugh, P. Augustijns, G. Van den Mooter, J. Van Humbeeck, J. Vanacken, V. V. Moshchalkov, J. Vermant and J. A. Martens, Chem. Commun., 2008, 47-49.

14 F. Müller, W. Peukert, R. Polke and F. Stenger, Int. J. Miner. Process., 2004, 74, S31-S41.

15 C. Urban and P. Schurtenberger, J. Colloid Interface Sci., 1998, 207, 150-158.

16 I. D. Block and F. Scheffold, Rev. Sci. Instrum., 2010, 81, 123107.

17 D. J. Pine, D. A. Weitz, P. M. Chaikin and E. Herbolzheimer, Phys. Rev. Lett., 1988, 60, 1134-1137.

18 G. Fortunato, A. Tenniche, L. Gottardo and R. Hufenus, Eur. Polym. J., 2014, 57, 75-82.

19 W. You, W. Yu and C. Zhou, Soft Matter, 2017, 13, 4088-4098.

20 F. J. Galindo-Rosales, P. Moldenaers and J. Vermant, Macromol. Mater. Eng., 2011, 296, 331-340.

21 K. A. Fröhlich, E. Mitrentsis, F. Clemens, B. Hoffmann, V. Michaud and T. Graule, Appl. Rheol., 2016, 26, 65050.

22 P. Cassagnau, Polymer, 2008, 49, 2183-2196.

23 J. Mewis and N. Wagner, Colloidal Suspension Rheology, Cambridge University Press, 2012.

24 J.-F. Le Meins, P. Moldenaers and J. Mewis, Ind. Eng. Chem. Res., 2002, 41, 6297-6304.

25 J. Mewis and N. J. Wagner, Adv. Colloid Interface Sci., 2009, 147-148, 214-227.

26 R. A. Lionberger and W. B. Russel, J. Rheol., 1994, 38, 18851908.

27 J. F. Brady, J. Chem. Phys., 1993, 99, 567-581.

28 J. Bergenholtz, F. Horn, W. Richtering, N. Willenbacher and N. J. Wagner, Phys. Rev. E, 1998, 58, R4088-R4091.

29 D. B. Genovese, Adv. Colloid Interface Sci., 2012, 171-172, 116.

30 J. Bergenholtz, N. Willenbacher, N. J. Wagner, B. Morrison, D. van den Ende and J. Mellema, J. Colloid Interface Sci., 1998, 202, 430-440.

31 C. Beenakker, Physica A, 1984, 128, 48-81.

32 J. W. Swan, E. M. Furst and N. J. Wagner, J. Rheol., 2014, 58, 307-337.

33 Z. Varga and J. W. Swan, J. Rheol., 2015, 59, 1271-1298.

34 A. Zaccone, H. H. Winter, M. Siebenbürger and M. Ballauff, J. Rheol., 2014, 58, 1219-1244.

35 R. de Rooij, D. van den Ende, M. H. G. Duits and J. Mellema, Phys. Rev. E, 1994, 49, 3038-3049.

36 J. van der Werff, C. de Kruif, C. Blom and J. Mellema, Phys. Rev. A, 1989, 39, 795-807.

37 B. Cichoki and B. U. Felderhof, Phys. Rev. A, 1991, 43, 54055411.

38 T. Shikata and D. S. Pearson, J. Rheol., 1994, 38, 601-616.
39 G. Colombo, S. Kim, T. Schweizer, B. Schroyen, C. Clasen, J. Mewis and J. Vermant, J. Rheol., 2017, 61, 1035-1048.

40 N. Willenbacher and C. Oelschlaeger, Curr. Opin. Colloid Interface Sci., 2007, 12, 43-49.

41 F. T. Adler, W. M. Sawyer and J. D. Ferry, J. Appl. Phys., 1949, 20, 1036-1041.

42 A. I. Romoscanu, M. B. Sayir, K. Häusler and A. S. Burbidge, Rheol. Acta, 2003, 42, 462-476.

43 G. Fritz, W. Pechhold, N. Willenbacher and N. J. Wagner, J. Rheol., 2003, 47, 303-319.

44 N. Willenbacher, C. Oelschlaeger, M. Schopferer, P. Fischer, F. Cardinaux and F. Scheffold, Phys. Rev. Lett., 2007, 99, 068302.

45 N. Willenbacher, J. S. Vesaratchanon, O. Thorwarth and E. Bartsch, Soft Matter, 2011, 7, 5777.

46 C. Oelschlaeger, M. Schopferer, M. Scheffold and N. Willenbacher, Langmuir, 2009, 25, 716-723.

47 M. Wang, H. H. Winter and G. K. Auernhammer, J. Colloid Interface Sci., 2014, 413, 159-166.

48 L. Kirschenmann and W. Pechhold, Rheol. Acta, 2002, 41, 362-368.

49 M. Cagnon and G. Durand, Phys. Rev. Lett., 1980, 45, 14181421.

50 M. Roth, M. D'Acunzi, D. Vollmer and G. K. Auernhammer, $J$. Chem. Phys., 2010, 132, 124702.

51 C. Clasen, B. P. Gearing and G. H. McKinley, J. Rheol., 2006, 50, 883-905.

52 J. L. Schrag, Trans. Soc. Rheol., 1977, 21, 399-413.

53 N. Ohl and W. Gleissle, J. Rheol., 1993, 37, 381-406.

54 G. K. Batchelor and J. T. Green, J. Fluid. Mech., 1972, 56, 401-427.

55 I. M. Krieger and T. J. Dougherty, Trans. Soc. Rheol., 1959, 3, 137-152.

56 R. A. Lionberger and W. B. Russel, in Advances in Chemical Physics, John Wiley and Sons, New York, 2000, ch. Microscopic theories of the rheology of stable colloidal dispersions, pp. 399-474.

57 A. Sierou and J. F. Brady, J. Fluid Mech., 2001, 448, 115-146.

58 Z. Cheng, J. Zhu, P. M. Chaikin, S.-E. Phan and W. B. Russel, Phys. Rev. E, 2002, 65, 041405.

59 A. Potanin, J. Colloid Interface Sci., 1993, 156, 143-152.

60 R. Wengeler and H. Nirschl, J. Colloid Interface Sci., 2007, 306, 262-273.

61 V. Nguyen, D. Rouxel and B. Vincent, Ultrason. Sonochem., 2014, 21, 149-153.

62 P. Akcora, S. K. Kumar, J. Moll, S. Lewis, L. S. Schadler, Y. Li, B. C. Benicewicz, A. Sandy, S. Narayanan, J. Ilavsky, P. Thiyagarajan, R. H. Colby and J. F. Douglas, Macromolecules, 2010, 43, 1003-1010.

63 R. Mangal, P. Nath, M. Tikekar and L. A. Archer, Langmuir, 2016, 32, 10621-10631. 